\title{
A Data Hiding Scheme for Grayscale Images Using a Square Function
}

\author{
Hyejin $\mathrm{Kwon}^{\dagger}$, Haemun Kim ${ }^{+\dagger}$, Soonja Kim ${ }^{++}$
}

\begin{abstract}
Many image hiding schemes based on least significant bit (LSB) transformation have been proposed. One of the LSB-based image hiding schemes that employs diamond encoding was proposed in 2008. In this scheme, the binary secret data is converted into base $n$ representation, and the converted secret data is concealed in the cover image. Here, we show that this scheme has two vulnerabilities: noticeable spots in the stego-image, i.e., a non-smooth embedding result, and inefficiency caused by rough $\mathrm{re}^{-} \mathrm{ad}^{-}$ justment of falling-off-boundary value and impractical base translation. Moreover, we propose a new scheme that is efficient and produces a smooth and high quality embedding result by restricting $n$ to power of 2 and using a sophisticated re-adjustment procedure. Our experimental results show that our scheme yields high quality stego-images and is secure against RS detection attack.
\end{abstract}

Key words: Image hiding, Steganography, hiding capacity, base conversion, modular arithmetic, RS detection attack

\section{INTRODUCTION}

Currently, the internet is the most-used channel among the various data communication channels. However, communication through the internet is vulnerable to many forms of attack, including eavesdropping and impersonation. These vulnerabilities cause security and privacy problems. To ensure security and privacy in communication via the internet, a cryptosystem is usually employed

※ Corresponding Author: Soon Ja Kim, Address: (702701) Daehakro 80, Bukgu, Daegu, Korea, TEL: +8253-950-5523, FAX : +82-53-950-8823, E-mail : snjkim@ ee.knu.ac.kr

Receipt date: Feb. 13, 2014, Revision date: Apr .1, 2014 Approval date: Apr .5, 2014

${ }^{+}$School of Electronics Engineering, Kyungpook National Univ.

(E-mail: heyjk90@gmail.com)

${ }^{++}$Department of Materials and Energy Engineering, Kyung woon Univ.

(E-mail: seadoor@hanmail.net)

${ }^{+++}$School of Electronics Engineering, Kyungpook National Univ.

※ This Research was supported by Kyungpook National University Research Fund, 2011.
[1-3]. A cryptosystem modifies the secret message into a series of meaningless bits so that an $\mathrm{ob}^{-}$ server (or eavesdropper) cannot understand the message. However, cryptosystems have a problem that results from the fact that encrypted messages are random bit strings: an observer can notice that the two entities communicate in secret $[1,4]$.

However, data hiding conceals the secret communication itself. Data hiding is a part of steganography with watermarking. Steganography is a set of methods that embed information into cover media. Usually, the cover medium is an image; in this case, we call the embedded result a stegoimage. One branch of image steganography(in short, steganography), watermarking, embeds a symbol to protect copyright or ownership. The hidden symbol in the embedded result does not have to be invisible, but it should tolerate many image manipulations and still contains the symbol. In another branch of steganography, image hiding, a secret message is embedded, and an observer should not detect the existence of a secret message in the stego-image, even if a large amount of secret information is hidden $[1,4,5-8,14]$. In fact, the size 
of the secret message and the imperceptibility of the secret data in the stego-image are two criteria of the embedded result; however, improving one requires sacrificing the other. Enhancing both criteria simultaneously is an open problem.

One method to easily obtain a stego-image is a simple LSB substitution scheme. In this scheme, the secret message is substituted for LSBs in each pixel. The number of substituted LSBs of each pixel depends on the length of the secret message and the size of the cover image. This scheme is based on the fact that changing the least significant bits (LSBs) has only a small effect on the whole image. However, an observer can easily detect the existence of secret data using steganalysis, such as $\mathrm{RS}$ detection attack $[4,5,9]$. In spite of flaws of simple LSB substitution scheme, it has inspired many researchers to study an image hiding scheme in the spatial domain.

In 2006, the EMD scheme [10] was proposed that greatly reduces the perceptibility of secret data in the embedded result by adding or subtracting 1 to the pixel value of the cover image. This image hiding scheme starts by translating secret data into a base $2 n+1$ representation, where $n$ is an embedding parameter. One digit of the secret data in the base $2 n+1$ numeral system is embedded in $n$ pixels. This scheme increases the quality of the embedded result by adding or subtracting 1 to the pixel value, but the total size of the secret message is limited.

To increase the allowed size of the secret message, Chao et al. proposed diamond encoding as an image hiding scheme [11]. However, we demonstrate that their scheme has poor efficiency and that the stego-image from their scheme has many gray stains, which allows an observer to detect the existence of secret data if the cover image has many pixels whose values are close to either 0 or 255 , as shown in Section $\Pi$. We propose a new image hiding scheme in Section III that provides a high quality embedded result efficiently using a square function. In Section IV, we present the $\mathrm{ex}^{-}$ perimental results and security analysis against RS detection attack[9]. Finally, we conclude in Section $\mathrm{V}$.

\section{REVIEW OF THE DIAMOND ENCOD- ING IMAGE HIDING METHOD}

In this section, we review the steganographic method with diamond encoding (in short, diamond scheme)[11]. In addition, we analyze the diamond scheme.

\subsection{Description of the diamond scheme}

In the diamond scheme, a grayscale cover image is divided into non overlapping blocks of two successive pixels. A block carries one digit in the base- $\left(2 k^{2}+2 k+1\right)$ numeral system, where $k$ is an embedding parameter. For simplicity, we let $2 k^{2}+2 k+1$ be represented by $l$. For each $k$, there exists a diamond function $f_{k}: Z_{256} \times Z_{256} \rightarrow Z_{l}$ where $f_{k}(p, q)=(2 k+1) p+q \bmod l$ and a diamond matching function $D_{k}: Z_{l} \rightarrow Z_{2 k+1} \times Z_{2 k+1}$. The diamond matching function $D_{k}$ is specified by a diamond shaped table, such as those in Fig. 1 and Fig. 2. Assuming that the coordinate of $0 \in Z_{l}$ is $(0,0) \in Z_{2 k+1} \times Z_{2 k+1}, D_{k}(a)$ is the coordinate of $a$ in the diamond shaped table $D_{k}$ for all $a \in Z_{l}$. For instance, $D_{2}(4)$ is $(1,-1)$.

The secret embedding procedure starts by $\mathrm{se}^{-}$ lecting the embedding parameter $k$. The embedding parameter $k$ is determined according to the length of the secret message $s$ and the size of the cover image $R \times C: k$ is the smallest integer that satisfies $\left\lfloor\frac{R \times C}{2} \log _{2}\left(2 k^{2}+2 k+1\right)\right\rfloor \geq|s|$. Then, the binary $\mathrm{se}^{-}$

cret message $s$ is translated into base $l$ representation $(s)_{l}, s \mapsto(s)_{l}\left(=s_{1}\left\|s_{2}\right\| \cdots\left\|s_{t}\right\| \cdots \| s_{m}\right)$, where $s_{t}$ is the $t$-th digit of $(s)_{l}, m$ is an integer that is less than half the image size $R \times C$ and $\|$ represents 


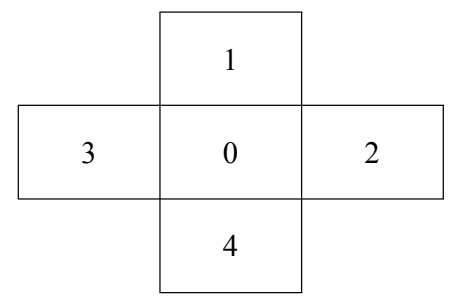

(a) $D_{1}$

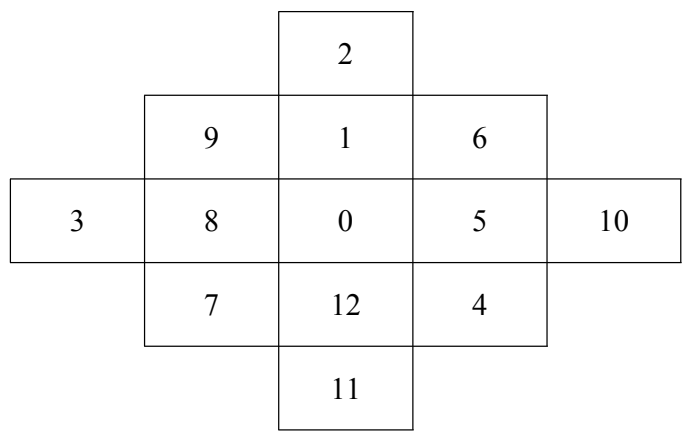

(c) $D_{2}$

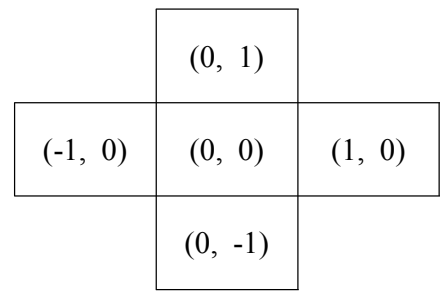

(b) The coordination of components in $D_{1}$

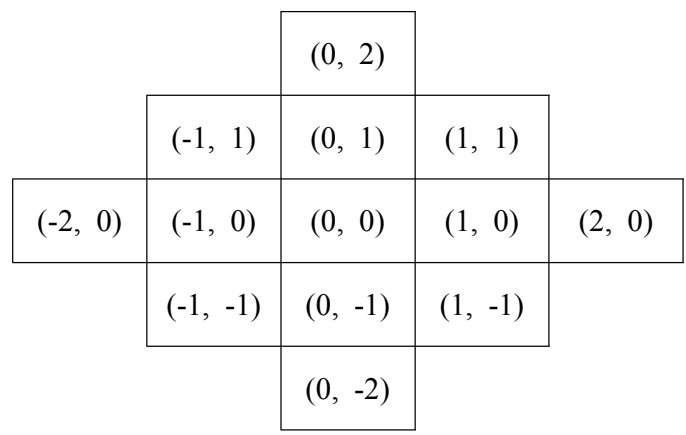

(d) The coordination of components in $D_{2}$

Fig. 1. The diamond matching function $D_{k}$ and its coordinates, where $k$ is 1 and is 2 .

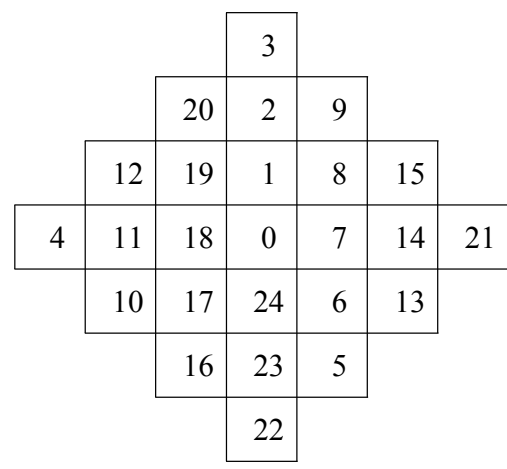

(a) $D_{3}$

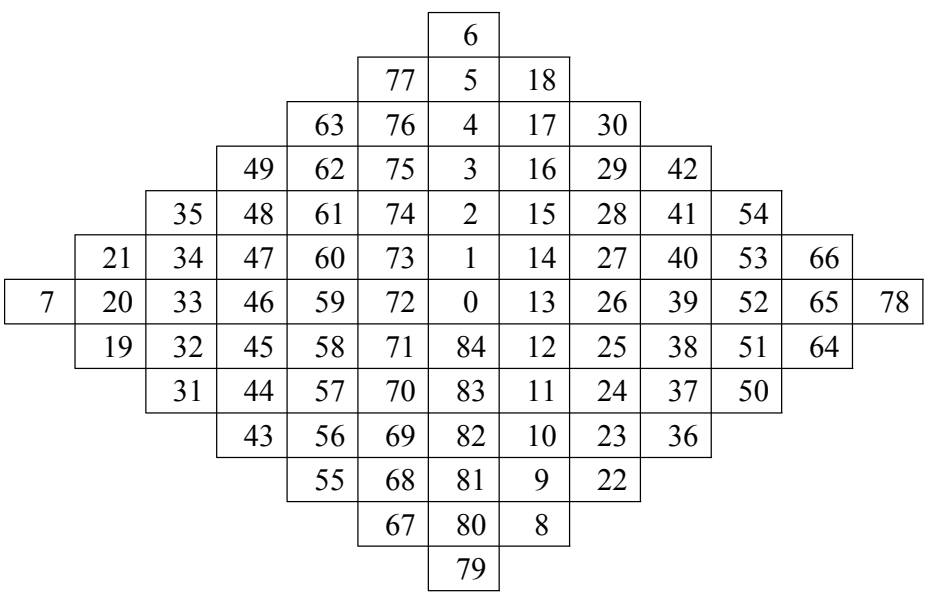

(b) $D_{6}$

Fig. 2. The diamond matching function $D_{k}$, where $k$ is 3 and is 6 .

concatenation. The value of the function $f_{k}$, $f_{k}\left(b_{t}\right)=f_{k}\left(p_{t}, q_{t}\right)=(2 k+1) p_{t}+q_{t} \bmod l$, is computed for every block $b_{t}$, where $b_{t}=\left(p_{t}, q_{t}\right)$ is the $t$-th block in the cover image and both $p_{t}, q_{t}$ are pixels. Then, the difference $d_{t}=s_{t}-f_{k}\left(b_{t}\right)$ is calculated for all $t$. Finally, the pair of stego-pixel values $\left(p_{t}{ }^{\prime}, q_{t}{ }^{\prime}\right)=$ $\left(p_{t}, q_{t}\right)+(a, b)$ are computed, where $(a, b)=D_{k}\left(d_{t}\right)$.
Here, some stego-pixels may not fall between 0 and 255 . We call these pixels the falling-off-boun-

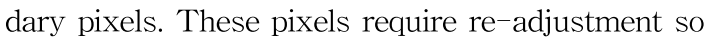
that they are included in $[0,255]$ : if the stego-pixel $x^{\prime}>255\left(\right.$ resp. $x^{\prime}<0$ ), then $x^{\prime}=x^{\prime}-l\left(\right.$ resp. $\left.x^{\prime}=x^{\prime}+l\right)$. The secret embedding procedure ends if all the falling-off-boundary pixels are re-adjusted. 
In the secret data extracting procedure, the stego-image is divided into non-overlapping blocks, as in the cover image. Then, the series of outputs of the diamond function for each block is the secret data $(s)_{l}$ in the base- $l$ numeral system. In other words, $f_{k}\left(b_{1}{ }^{\prime}\right)\left\|f_{k}\left(b_{2}{ }^{\prime}\right)\right\| \cdots\left\|f_{k}\left(b_{t}{ }^{\prime}\right)\right\| \cdots \| f_{k}\left(b_{m}{ }^{\prime}\right)$ is the secret message that is converted into base $l$ representation, where $b_{t}^{\prime}$ is the $t$-th block of stego-image and $m \leq \frac{R \times C}{2}$. Thus, the binary secret message can be obtained by translating the series of $f_{k}\left(b_{t}^{\prime}\right)$ into base-2 representation.

Example. We give an example of producing a stego-block according to the diamond scheme. Assuming that the embedding parameter $k$ is 3 , then we determine that $l$ is 25 . We let the $t$-th digit $s_{t}$ of the secret message in the base- 25 numeral system be $6 \in Z_{25}$, and the $t$-th block $b_{t}=\left(p_{t}, q_{t}\right)$ of the cover image be $(200,190)$. The diamond function value of $b_{t}$ is $f_{3}\left(b_{t}\right)=f_{3}(200,190)=7 \times 200+190 \bmod$. $25=15$.Then, $d_{t}=s_{t}-f_{3}\left(b_{t}\right) \bmod l=6-15 \bmod 25=16$. The output of the diamond matching function $D_{3}\left(d_{t}\right)$ is $(-1,-2)$, as shown in Fig. 2.(a). Thus, the $t$-th block of the stego-image is $b_{t}+D_{3}\left(d_{t}\right)=$ $(199,188)$. The secret digit $s_{t}$ concealed in $t^{- \text {th stego- }}$ block is extracted by the diamond function as fol-

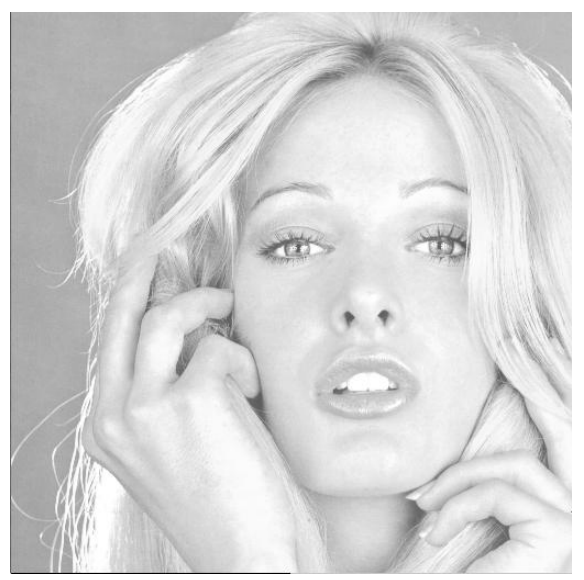

(a) Cover image : Tiffany lows: $s_{t}=f_{k}\left(b_{t}^{\prime}\right)=f(199,188)=7 \times 199+188 \bmod 25=6$.

\subsection{Analysis of the diamond scheme}

This section shows the two drawbacks of the diamond scheme : a non-smooth embedded result and inefficiency.

\subsubsection{Nonsmooth stego-image}

The diamond scheme solves the falling-offboundary problem by simply adding or subtracting $l$ to the falling-off-boundary pixel value. This re-adjustment procedure is simple to apply, but the difference between the original cover pixel and stego-pixel is remarkable when $l$ is large. This large difference makes the embedded result look non-smooth.

For example, let $k=3(l=25), s_{t}=9, b_{t}=(253,254)$. Then, stego-block $b_{t}{ }^{\prime}$ is $(254,256)$. This block requires re-adjustment because the second pixel of this block overflows the boundary value 255 . The re-adjusted block is $(254,256)-(0,25)=(254,231)$. As a result, the difference between the original block and the stego-block is $(1,23)$, which is a significant difference that causes the embedded result to have a perceptible spot.

Fig. 3. shows a non-smooth stego-image when

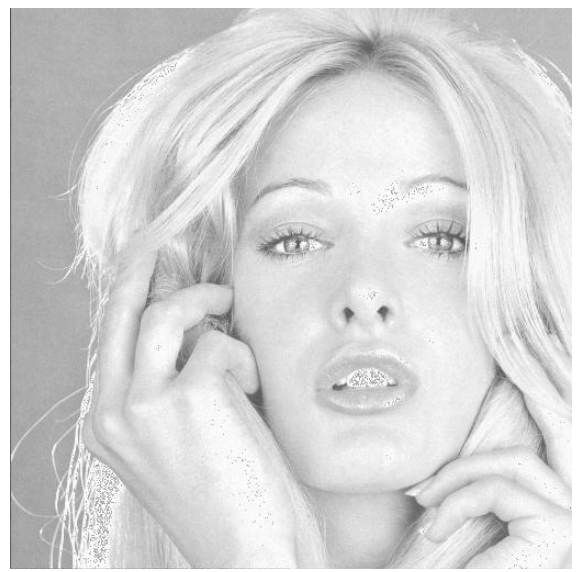

(b) Stego-image generated by the diamond scheme with $|s|=777350$ bits

Fig. 3. Non-smoothness of a stego-image generated by the diamond scheme. 
the secret data $s$ is a series of random bits with $|s|=777350$, which is less than $3 \times R \times C$, where $R \times C$ is the image size. Compared with the cover image, the embedded result has many perceptible spots in Tiffany's hair and teeth so that the image is not smooth. Even if the peak of the signal-to-noise ratio (PSNR) that is employed to estimate the quality of the stego-image is pretty high $(35.21 \mathrm{~dB})$, we can still detect the existence of a secret message[1, 11,12]. In short, the embedded result looks nonsmooth when the cover image has many pixels whose values are in the neighborhood of a boundary value $(0$ or 255$)$ and $l$ is large.

In the diamond scheme, binary secret data is converted to its representation in base- $l$. Because $l$ is not a power of 2 , the base conversion is not easy when the secret data is large. In fact, the time required to convert $|s|$-bit secret data into base- $l$ representation is $O\left(|s|^{2}\right)$ [13]. Moreover, the naïve conversion procedure has difficulty translating the base, even if the secret data has about 200,000 bits.

\subsubsection{Inefficiency}

In the diamond scheme, binary secret data is converted to its representation in base $-l$. Because $l$ is not a power of 2 , the base conversion is not easy when the secret data is large. In fact, the time required to convert $|s|$-bit secret data into base- $l$ representation is $O\left(|s|^{2}\right)$ [13]. Moreover, the naïve conversion procedure has difficulty translating the base, even if the secret data has about 200,000 bits.

\section{PROPOSED SCHEME WITH SQUARE FUNCTION}

In this section, we propose an image hiding scheme that employs a square function to address two problems of the diamond scheme. In order to solve the non-smoothness of the stego-image, we employ a refined re-adjustment step for fallingoff-boundary pixels rather than the rough $\mathrm{re}^{-} \mathrm{ad}^{-}$ justment procedure, such as adding or subtracting a relatively large number $l$. Moreover, to solve the inefficiency, we set the base $l$ to a power of 2 .

Notations. The followings are notations used in this paper.

$k$ : the embedding parameter

$l: 2^{2 k}$

$s \quad$ : the secret data

$|s|$ : the length of secret data

$(s)_{l}$ : the secret data translated into base- $l$ representation

$s_{t}:$ the $t$-th digit of $(s)_{l}$

$b_{t}:$ the $t$-th block of an image. $b_{t}=\left(p_{t}, q_{t}\right), p_{t}$ and $q_{t}$ are two consecutive pixels. In particular, we leave $b^{\prime}{ }_{t}$ for the $t$-th block of the stego-image.

$d_{t}$ : the difference between $s_{t}$ and $B_{k}\left(b_{t}\right)$

$B_{k}$ : for each $k$, there is a block function $B_{k}: Z_{256} \times Z_{256} \rightarrow Z_{l}$, where $B_{k}(p, q)=2^{k} p+q \bmod l$

$S_{k}$ : for each $k$, there is a square function $S_{k}: Z_{l} \rightarrow Z_{2^{k}} \times Z_{2^{k}}$, where $S_{k}$ is the inverse function of $\left.B_{k}\right|_{Z_{z^{*}} \times Z_{2^{*}}}$, with the restriction of $B_{k}: Z_{256} \times Z_{256} \rightarrow Z_{l}$ to $Z_{2^{k}} \times Z_{2^{k}}$. In other words, $S_{k}^{-1}(a, b)=2^{k} a+b \bmod l$, for all $(a, b) \in Z_{2^{k}} \times Z_{2^{k}}$. This function is explicitly represented by a table like those in Fig. 4.(a), (b), and (c). We let the coordinate of $0 \in Z_{l}$ be $(0,0) \in Z_{2^{k}} \times Z_{2^{k}}$. Then, $S_{k}(n)$ is the coordinate of $n$ in the table. For example, $S_{2}(12)$ is $(-1,0)$, as shown in Fig. 4(a) and (b).

\subsection{Data embedding procedure}

In proposed scheme, a grayscale cover image is divided into non-overlapping blocks, and one block consists of two consecutive pixels, as in the diamond scheme. Furthermore, one digit of the secret data that is translated into base ${ }^{-2 k}$ representation can be hidden in each block. In this section, we describe proposed data embedding procedure in detail.

\subsubsection{Setup phase}

- Cover image division : the cover image with 


\begin{tabular}{|c|c|c|c|}
\hline 14 & 2 & 6 & 10 \\
\hline 13 & 1 & 5 & 9 \\
\hline 12 & 0 & 4 & 8 \\
\hline 11 & 15 & 3 & 7 \\
\hline
\end{tabular}

(a) $S_{2}$

\begin{tabular}{|l|l|l|l|l|l|l|l|}
\hline 44 & 52 & 60 & 4 & 12 & 20 & 28 & 36 \\
\hline 43 & 51 & 59 & 3 & 11 & 19 & 27 & 35 \\
\hline 42 & 50 & 58 & 2 & 10 & 18 & 26 & 34 \\
\hline 41 & 49 & 57 & 1 & 9 & 17 & 25 & 33 \\
\hline 40 & 48 & 56 & 0 & 8 & 16 & 24 & 32 \\
\hline 39 & 47 & 55 & 63 & 7 & 15 & 23 & 31 \\
\hline 38 & 46 & 54 & 62 & 6 & 14 & 22 & 30 \\
\hline 37 & 45 & 53 & 61 & 5 & 13 & 21 & 29 \\
\hline
\end{tabular}

(c) $S_{3}$

\begin{tabular}{|c|l|l|l|}
\hline$(-1,2)$ & $(0,2)$ & $(1,2)$ & $(2,2)$ \\
\hline$(-1,1)$ & $(0,1)$ & $(1,1)$ & $(2,1)$ \\
\hline$(-1,0)$ & $(0,0)$ & $(1,0)$ & $(2,0)$ \\
\hline$(-1,-1)$ & $(0,-1)$ & $(1,-1)$ & $(2,-1)$ \\
\hline
\end{tabular}

(b) The coordinates of components in $S_{2}$

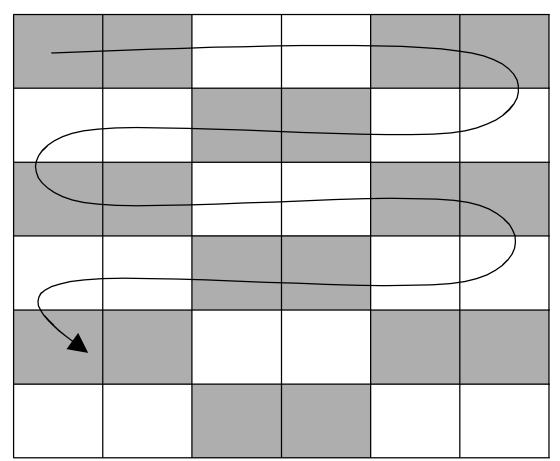

(d) Non-overlapping blocks of two consecutive pixels and the order of blocks

Fig. 4. The square function $S_{2}$ and its coordinates, the square function $S_{3}$, and construction of blocks and the direction of the embedding progress

$R \times C$ pixels is divided into non-overlapping blocks of two pixels, as shown in Fig. 4(d). Simply we represent the cover image as series of blocks $b_{1}\left\|b_{2}\right\| \cdots\left\|b_{t}\right\| \cdots \| b_{\frac{R \times C}{2}}$.

- Determining the embedding parameter $k:$ the embedding parameter $k$ depends on the image size $R \times C$ and the length of the secret message $|s|$. In detail, $k$ is the smallest integer that satisfies $k \geq \frac{|s|}{R \times C}$.

- Base translation : the secret message $s$ should be converted into $(s)_{l}$. In other words, the base of $s$ must be translated into $l$.

\subsubsection{Encoding phase}

The embedding phase is described below. It is similar to the diamond scheme, except for the re-adjustment step.

a. Find the block function value $B_{k}\left(b_{t}\right)$ for each $b_{t}$.

b. Compute the difference $d_{t}=s_{t}-B_{k}\left(b_{t}\right) \bmod l$ for every $t$

c. Find $S_{k}\left(d_{t}\right)=(a, b)$ for every $t$.

d. Compute the pre-stego-block $b_{t}^{\prime}=b_{t}+S_{k}\left(d_{t}\right)=\left(p_{t}, q_{t}\right)+(a, b)$ for every $\mathrm{t}$.

e. The pre-stego-block may contain the falling-off-boundary pixels; thus, the pre--stegoblock should be re-adjusted as follows.

i. If $p^{\prime}{ }_{t} \leq 0$ and $q_{t}^{\prime}<0$, then $p_{t}^{\prime}=p_{t}{ }^{\prime}+2^{k}-1$, $q_{t}^{\prime}=q_{t}^{\prime}+2^{k}$.

ii. If $p^{\prime}{ }_{t}<0$ and $0 \leq q_{t}{ }^{\prime} \leq 255$, then $p_{t}{ }^{\prime}=p_{t}{ }^{\prime}+2^{k}$, $q_{t}^{\prime}=q_{t}^{\prime} \cdot 1$

iii. If $p^{\prime}{ }_{t}<0$ and $q_{t}^{\prime}>255$, then $p_{t}{ }^{\prime}=p_{t}{ }^{\prime}+2^{k}+1$, $q_{t}^{\prime}=q_{t}^{\prime}-2^{k}$.

iv. If $0<p_{t}{ }^{\prime} \leq 256$ and $q_{t}{ }^{\prime}<0$, then $p_{t}{ }^{\prime}=p_{t}{ }^{\prime}-1$, $q_{t}^{\prime}=q_{t}^{\prime}+2^{k}$.

v. If $0 \leq p_{t}{ }^{\prime}<255$ and $q_{t}^{\prime}>255$, then $p_{t}^{\prime}=p_{t}{ }^{\prime}+1$, 


$$
q_{t}^{\prime}=q_{t}^{\prime}-2^{k}
$$

vi. If $p^{\prime}{ }_{t}>255$ and $0 \leq q_{t}{ }^{\prime} \leq 255$, then $p_{t}{ }^{\prime}=p_{t}{ }^{\prime}-2^{k}$, $q_{t}^{\prime}=q_{t}^{\prime}$.

vii. If $p_{t}^{\prime}>256$ and $q_{t}^{\prime}<0$, then $p_{t}^{\prime}=p_{t}^{\prime}-2^{k}-1$, $q_{t}^{\prime}=q_{t}^{\prime}+2^{k}$.

viii. If $p_{t}^{\prime} \geq 255$ and $q_{t}^{\prime}>255$, then $p_{t}^{\prime}=p_{t}^{\prime}-2^{k}+1$, $q_{t}^{\prime}=q_{t}^{\prime}-2^{k}$.

ix. Otherwise, the stego-block is the same as the pre-stego-block.

Because the block function value of the stegoblock includes information about the secret message, block function value should not be changed after the block is re-adjusted. One solution is to set, where is a falling-off-boundary pixel, as in the diamond scheme. However, there is large gap between the cover image and stego-image with this rough re-adjustment step; thus, it allows the secret message to be perceived. The proposed sophisticated re-adjustment step employs a value less than , rather than employing; as a result, we reduce the difference between the cover image and the stego-image. Moreover, there is no difference in the block function value before and after the re-adjustment step.

$\mathrm{f}$. The stego-image is a composition of stegoblocks. Namely, the stego-image is $b_{1}{ }^{\prime}\|\cdots\| b_{t}{ }^{\prime}\|\cdots\| b_{\frac{R \times C}{2}}{ }^{\prime}$.

\subsubsection{Data extraction procedure}

Extraction of the secret message from the stego-image is simpler than embedding.

a. The stego-image is divided into non-overlapping blocks of two pixels, as in the embedding procedure. As a result, the stego-image is represented as $b_{1}^{\prime}\|\cdots\| b_{t}^{\prime}\|\cdots\| b_{\frac{R \times C^{\prime}}{2}}$.

b. Find the block function value $B_{k}\left(b_{t}^{\prime}\right)$ for each $b_{t}^{\prime}$.

c. Concatenate all block function values, and then obtain the pre-secret message $(s)_{l}$. Namely, the pre-secret message $(s)_{l}$ is $B_{k}\left(b_{1}{ }^{\prime}\right)\|\cdots\| B_{k}\left(b_{t}{ }^{\prime}\right)\|\cdots\| B_{k}\left(b_{\frac{R \times C}{2}}{ }^{\prime}\right)$.

d. The secret message $s$ is obtained by converting the pre-secret message $(s)_{l}$ to binary data.

Note. The following illustrates why $B_{k}\left(b_{t}{ }^{\prime}\right)=s_{t}$. $B_{k}\left(b_{t}{ }^{\prime}\right)=2^{k} p_{t}{ }^{\prime}+q_{t}{ }^{\prime} \bmod l=2^{k}\left(p_{t}+a\right)+\left(q_{t}+b\right) \bmod l$ $=2^{k} p_{t}+q_{t}+2^{k} a+b \bmod l=B_{k}\left(b_{t}\right)+S_{k}^{-1}(a, b)$ $=B_{k}\left(b_{t}\right)+d_{t}=s_{t}$.

Example. We give an example of producing a stego-block with proposed scheme. Assuming that the embedding parameter $k$ and $l$ are 2 and 16, respectively. We let the $t$-th digit of secret data $s_{t}$ be $12 \in Z_{16}$, and the $t$-th block $b_{t}=(253,254)$. Then the block function value $B_{2}(253,254)$ is $2^{2} \times 253+254 \bmod 16=2$. And $d_{t}=s_{t}-B_{2}\left(b_{t}\right) \bmod l=10$, $S_{2}\left(d_{t}\right)=S_{2}(10)=(2,2)$ as shown in Fig. 4.(a) and (b). Thus the $t$-th pre-stego-block $b_{t}^{\prime}$ is $b_{t}+S_{2}\left(d_{t}\right)=(255,256)$. This block requires to be re-adjusted because the second pixel of the block overflows the boundary value 255 . By e.viii of embedding phase, the final stego-block $b_{t}^{\prime}$ is $(252,252)$. The extraction procedure is $s_{t}=B_{2}\left(b_{t}{ }^{\prime}\right)$ $=2^{2} \times 252+252 \bmod 16=12$.

\section{EXPERIMENTAL RESULTS}

In this section, we present our experimental results, including a quality comparison with other schemes and security analysis against RS detection attack. We experimented with the wellknown 8-bit grayscale images "Couple", "Zelda", "Lena", "Tiffany", "F16", "Baboon", and "Pepper" in Fig. 5, 6, and 7; the size of first two images is $256 \times 256$, and the others are $512 \times 512$. Moreover, we performed the experiment with MATLAB, and the secret message was generated by the pseudo-random function in MATLAB. 


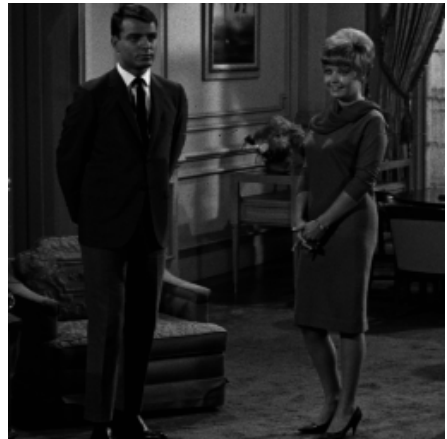

(a) Couple

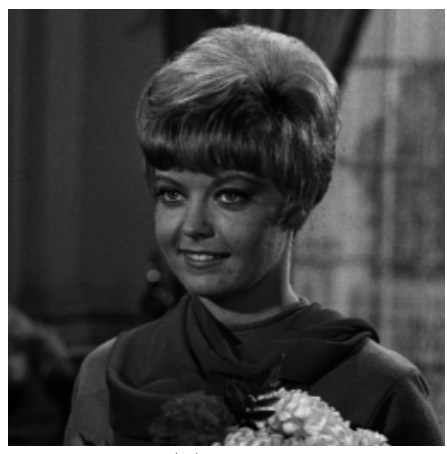

(d) Zelda

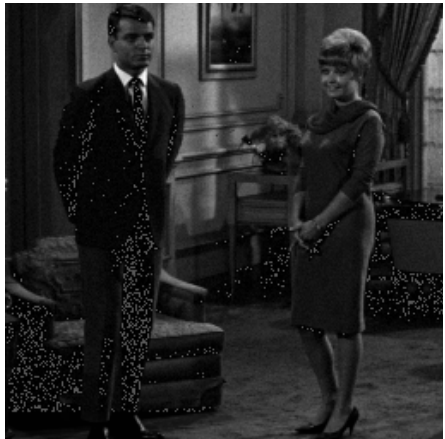

(b) Diamond scheme

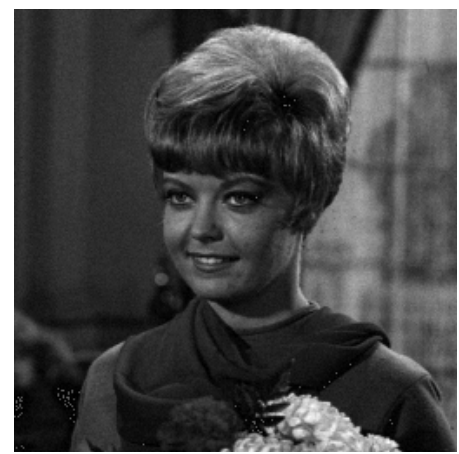

(e) Diamond scheme

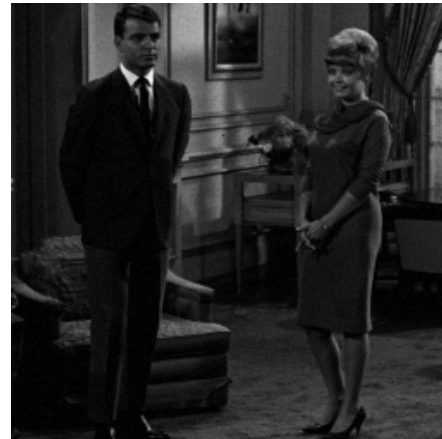

(c) Proposed scheme

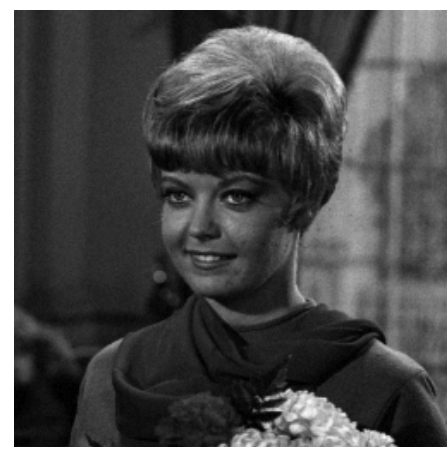

(f) Proposed scheme

Fig. 5. The cover images and stego-images with $256 \times 256$ images generated by the diamond scheme and the proposed scheme where $|s|=196608$ bits.

\subsection{Quality and capacity}

We employ peaks of the signal-to-noise ratio (PSNR) to estimate the quality of the stegoimages. The capacities and relative PSNRs for various test images are shown in Table 1 and Table. 2. Table 1 and Table 2, respectively, represent the PSNRs when the capacity is $2 \times R \times C$ bits

Table 1. Comparison of PSNR with simple LSB substitution scheme, the diamond scheme, and ours when $|s|=2 \times R \times C$, where $R \times C$ is the image size

\begin{tabular}{|c|c|c|c|}
\hline \multirow{2}{*}{ Test image } & \multicolumn{3}{|c|}{ PSNR(dB) } \\
\cline { 2 - 4 } & Simple LSB & Diamond & Proposed \\
\hline \hline Couple & 44.26 & 39.51 & 46.22 \\
Zelda & 44.19 & 44.69 & 46.36 \\
Lena & 44.15 & 45.50 & 46.38 \\
Tiffany & 44.12 & 43.96 & 46.33 \\
F16 & 44.17 & 45.60 & 46.36 \\
Baboon & 44.15 & 45.59 & 46.36 \\
Pepper & 44.16 & 45.60 & 46.37 \\
\hline
\end{tabular}

and $3 \times R \times C$ bits with an image size of $R \times C$.

The tables show that our scheme provides consistent PSNRs over various images. On the other hand, we find that the PSNRs of stego-images generated by the diamond scheme are inconsistent and depend on the cover images. In detail, the diamond scheme and the proposed method yield sim-

Table 2. Comparison of PSNR with simple LSB substitution scheme, the diamond scheme, and ours when $|s|=3 \times R \times C$ with an image size of $R \times C$

\begin{tabular}{|c|c|c|c|}
\hline \multirow{2}{*}{ Test image } & \multicolumn{3}{|c|}{ PSNR $(\mathrm{dB})$} \\
\cline { 2 - 4 } & Simple LSB & Diamond & Proposed \\
\hline \hline Couple & 37.97 & 25.49 & 40.32 \\
Zelda & 37.97 & 35.33 & 40.71 \\
Lena & 37.91 & 39.12 & 40.73 \\
Tiffany & 37.92 & 33.02 & 40.64 \\
F16 & 37.98 & 39.92 & 40.73 \\
Baboon & 37.94 & 39.92 & 40.73 \\
Pepper & 37.93 & 39.67 & 40.72 \\
\hline
\end{tabular}


ilar outputs, except "Couple", "Zelda", and "Tiffany": the suit of a man in Fig. 5.(b) and the hair and the cloth in Zelda in Fig. 5.(e) have many gray stains. Moreover, the teeth and hair of Tiffany in Fig. 6.(e) have many gray spots. These three cover images have multiple pixels whose values are close to either 0 or 255. As cited in Section П.2.A, the diamond scheme provides a poor embedding result when the size of the embedded message is large and the cover image have many neighborhoods of pure white or pure black (pixel values of 255 and 0 , respectively). In the meantime, our scheme yields reasonable embedding results irrespective of the images.

\subsection{Security: RS detection attack}

This section shows that our scheme is secure against RS detection attack[9]. The RS detection attack was proposed by Fridrich et al. and detects stego-images. In their paper, a discrimination function $f$ is defined that estimates the smoothness of a pixel group, an invertible operation flipping $F$, and a row vector of length $n$ mask $M$. To determine whether or not an image is a stego-image, the im-

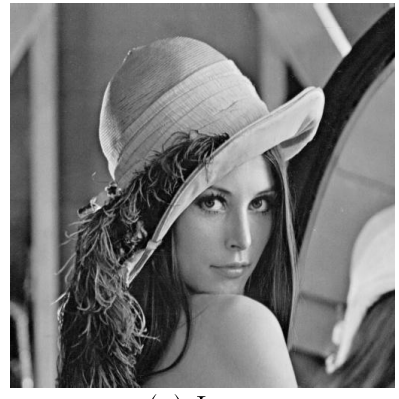

(a) Lena

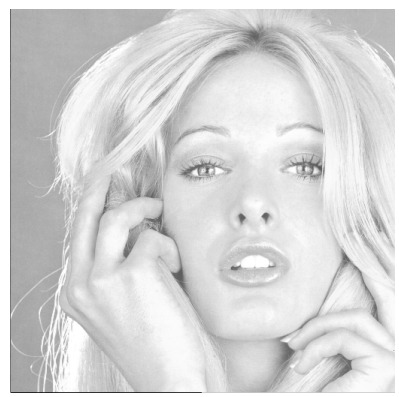

(d) Tiffany

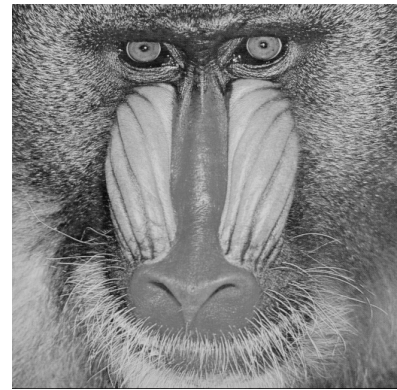

(g) Baboon

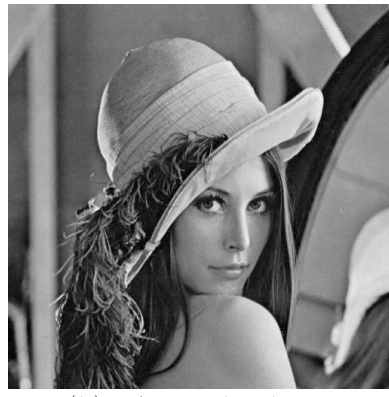

(b) Diamond scheme

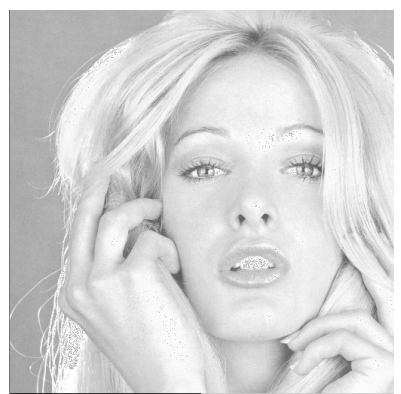

(e) Diamond scheme

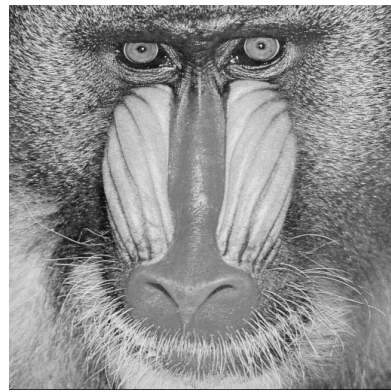

(h) Diamond scheme

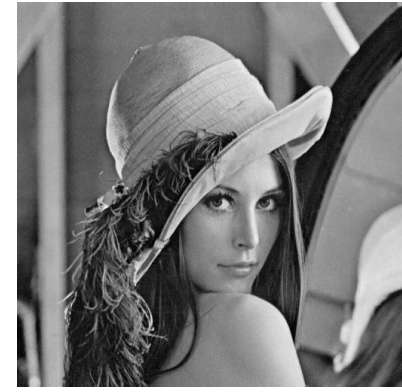

(c) Proposed scheme

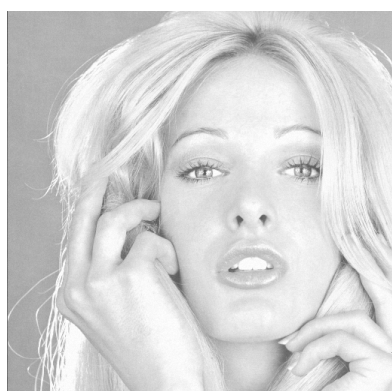

(f) Proposed scheme

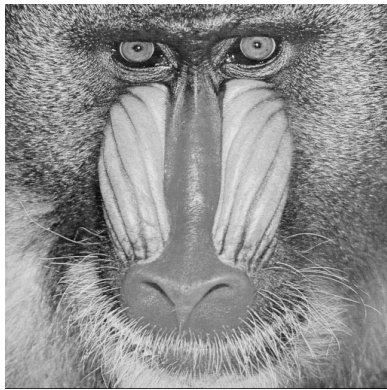

(i) Proposed scheme

Fig. 6. The cover images and stego-images with $512 \times 512$ images generated by the diamond scheme and the proposed scheme where $|s|=786432$ bits. 


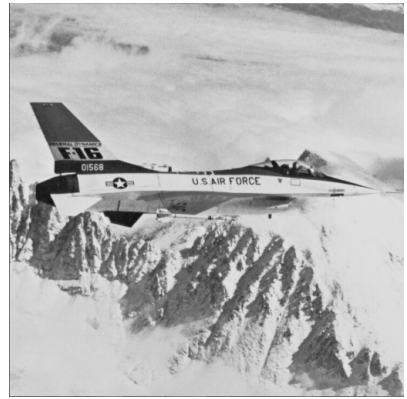

(a) F16

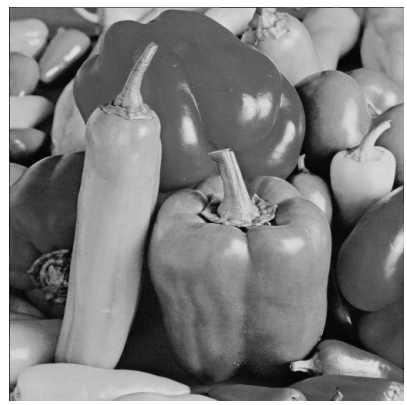

(d) Pepper

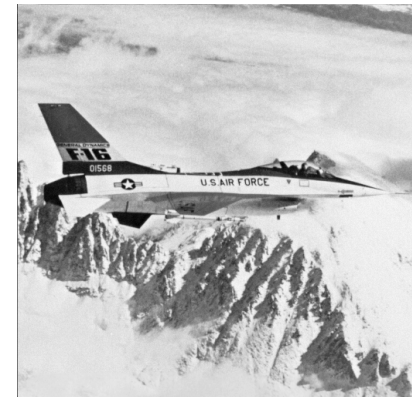

(b) Diamond scheme

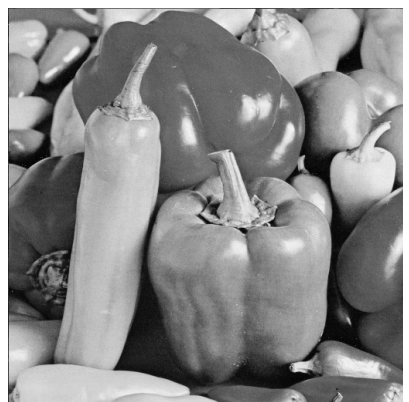

(e) Diamond scheme

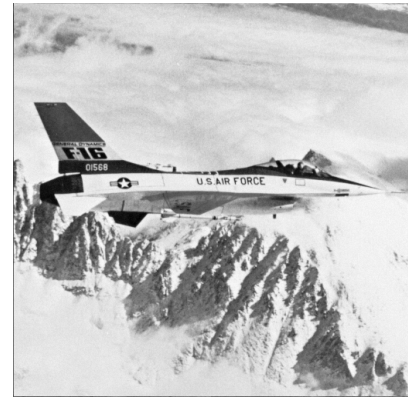

(c) Proposed scheme

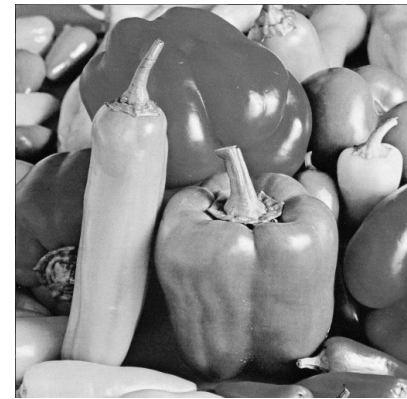

(f) Proposed scheme

Fig. 7. The cover images and stego-images with $512 \times 512$ images generated by the diamond scheme and the proposed scheme where $|s|=786432$ bits.

age is divided into disjoint groups of $n$ adjacent pixels. Furthermore, $f(G)$ is compared with $f\left(F_{M}(G)\right)$, where $G$ is a pixel group and $F_{M}(G)$ is flipped $G$ with mask $M$. If $f(G)$ is larger(or smaller) than $f\left(F_{M}(G)\right)$, the pixel group $G$ is defined as a regular group $R$ (or singular group $S$ ). Otherwise, it is defined as an unusable group $U$. The value of $R_{M}$ (or $S_{M}$ and $U_{M}$ ) is the number of regular(or singular and unusable) groups with mask $M$. Finally, the image is judged to be a stego-image if it does not satisfy the following properties: $R_{M} \cong R_{-M}$ and $S_{M} \cong S_{-M}$.

We subjected the stego-images generated by our scheme to an RS detection attack with $f(G)=f\left(x_{1}, x_{2}, \cdots, x_{n}\right)=\sum_{i=1}^{n-1}\left|x_{i+1}-x_{i}\right| \quad$ as a discrimination function and [ $\left[\begin{array}{llll}0 & 1 & 1 & 0\end{array}\right]$ as the mask $M$. Fig. 8. is one attack result when the cover image is "Lena", and we obtained similar results for the other images.

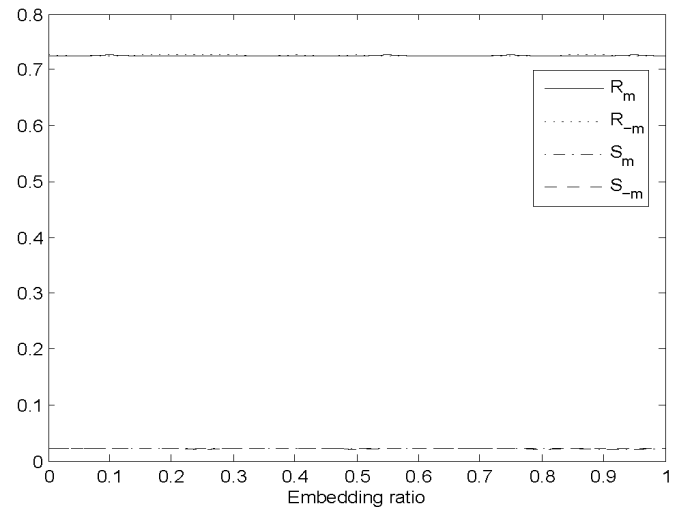

Fig. 8. Security of proposal scheme against RS attack.

\section{CONCLUSION}

To secure communication, research on an image hiding scheme that hides the communication itself in the images has been proposed in addition to cryptography. In image hiding, the capacity and imperceptibility of secret data in the stego-image 
are two important barometers of the quality of the scheme. Many researchers have proposed LSBbased image hiding schemes to conceal a large quantity of secret data while maintaining the imperceptibility of the secret data in the stego-image. Among the image hiding schemes, we reviewed the diamond scheme proposed by Chao et al. and revealed two drawbacks of the diamond scheme: poor embedding results for images that have many pixels whose values are close to 0 or 255 when there is a large quantity of secret data and inefficiency caused by base conversion. We proposed a new image hiding scheme that employs a square function. In addition, the proposed scheme employs a sophisticated re-adjustment procedure for falling-off-boundary pixels and efficient base conversion. In detail, one digit in the base $2^{2 k}$ numeral system is embedded in two pixels. Moreover, we showed that the proposed scheme provides higher quality results than the diamond scheme according to the experimental results.

\section{REFERENCES}

[1] Y. H. Yu, C. C. Chang, and I. C. Lin, "A New Steganographic Method for Color and Grayscale Image Hiding," Computer Vision and Image Understanding, Vol. 107, No. 3, pp. 183-194, 2007.

[2] C. C. Change and H. W. Tseng, "A Steganographic Method for Digital Images using Side Match," Pattern Recognition Letters, Vol. 25, No. 12, pp. 1431-1437, 2004.

[ 3 ] C. C. Lin and N. L. Hsueh, "A Lossless Data Hiding Scheme based on Three-pixel Block Differences," Pattern Recognition, Vol. 41, No. 4, pp. 1415-1425, 2008

[4] R. Z. Wang, C. F. Lin, and J. C. Lin, "Image Hiding by Optimal LSB Substitution and Genetic Algorithm," Pattern Recognition, Vol. 34, No. 3, pp. 671-683, 2001.

[5] T. Morkel, J. H. P. Eloff, and M. S. Olivier,
"An Overview of Image Steganography," Information and Computer Security Architecture (ISSA), pp. 1-11, 2005.

[6] C. L. Liu and S. R. Liao, "High-performance JPEG Steganography using Complementary Embedding Strategy," Pattern Recognition, Vol. 41, No. 9, pp. 2945-2955, 2008.

[7] C. C. Chang, C. Y. Lin, and Y. Z. Wang, "New Image Steganographic Methods using Runlength Approach," Information Sciences, Vol. 176, No. 22, pp. 3393-3408, 2006.

[8] D. C. Wu and W. H. Tsai, "A Steganographic Method for Images by Pixel-value Differencing," Pattern Recognition Letters, Vol. 24, No. 9, pp. 1613-1626, 2003.

[9] J. Fridrich, M. Goljan, and R. Du, "Reliable Detection of LSB Steganography in Color and Grayscale Images," Proceeding of the 2001 Workshop on Multimedia and Security: New Challenges, pp. 27-30, 2001.

[10] X. Zhang and S. Wang, "Efficient Steganographic Embedding by Exploiting Modification Direction," IEEE Communications Letters, Vol. 10, No. 11, 781-783, 2006.

[11] R. M. Chao, H. C. Wu, C. C. Lee, and Y. P. Chu, "A Novel Image Data Hiding Scheme with Diamond Encoding," European Association for Signal Processing (EURASIP) Journal on Information Security, Vol. 2009, No. 1, pp. 1-9, 2009

[12] C. C. Lin, W. L. Tai, and C. C. Chang, "Multilevel Reversible Data Hiding based on Histogram Modification of Difference Images," Pattern Recognition, Vol. 41, No. 12, 35823591, 2008.

[13] N. Koblitz, A Course in Number Theory and Cryptography, Springer, New York, 1994.

[14] D. Kim, K. Yoo, " A novel Reversible Data Hiding Scheme based on Modulo Operation and Histogram Shifting," Journal of Korea Multimedia Society, Vol. 15, No. 5, pp. 639650, 2012 


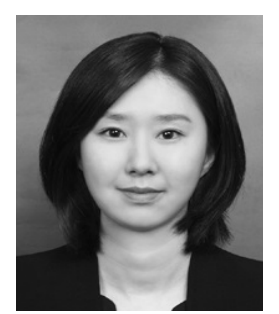

\section{Hyejin Kwon}

Feb. 2007: Kyungpook National University, Dept. of Mathematics, B.A.

Feb. 2009: Kyungpook National University, Dept. of Information Security, M.S.

Feb. 2009 : Kyungpook National University, Dept. of Electronics Engineering, $\mathrm{PhD}$ Candidate

Research Interest: Information security, Cryptography, Network security, Steganography

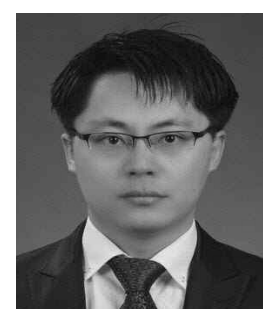

\section{Haemun Kim}

Feb. 2002: Kyungpook National University, Dept. of Electronics Engineering, B.S

Feb. 2004: Kyungpook National University, Dept. of Electronics Engineering, M.S..

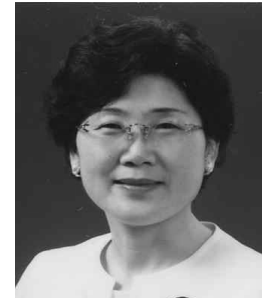

Soonja Kim

Feb. 1975: Kyungpook National University, Dept. of Mathematics Education, B.S.

Feb. 1977: Kyungpook National University, Dept. of Mathematics Education, M.S.

Feb. 1988: Keimyung University, Dept. of Mathematics, $\mathrm{PhD}$.

Apr. 1993 : Kyungpook National University, Dept. of Electronics Engineering, Professor

Research Interest: nformation security, Cryptography, Network security

Feb. 2005: Kyungpook National University, Dept. of Electronics Engineering, $\mathrm{PhD}$ Candidate

Research Interest: Information security, Cryptography, Network security, Steganography 